\title{
An Extension of Proxy Mobile IPv6 for Reducing Handover Latency and Packet Loss using Transient Binding
}

\author{
L.K.Indumathi \\ National College of Engineering \\ Maruthakulam \\ Tirunelveli, Tamil Nadu \\ India
}

\author{
D.Shalini Punitha Vathani, \\ PhD. \\ Government College of \\ Engineering \\ Tirunelveli \\ Tamil Nadu \\ India
}

\author{
Sheryl Radley \\ PhD scholar \\ Government College of Eng \\ Tamil Nadu \\ India
}

\begin{abstract}
Proxy Mobile IPv6 (PMIPv6) is a protocol for building a common and access technology independent of mobile core networks, accommodating various access technologies such as WiMAX, 3GPP, 3GPP2 and WLAN based access architectures. Transient Binding is a mechanism applicable to the mobile node's inter-MAG handover while using a single interface or different interfaces. Proxy Mobile IPv6 (or PMIPv6, or PMIP) is a network-based mobility management protocol This paper proposes an improvement of Proxy Mobile Ipv6 in the aspect of reducing packet loss and hand over latency. Proxy Mobile IPv6 also implements additional features not present in IPv4. This paper proposes an improvement in the Proxy Mobile IPv6.The extension of Proxy Mobile IPv6 with transient binding will support multihoming and optimizes the handover. Here the handover problem in multihoming is reduced by transient binding by using modified Local Mobility Anchor (m-LMA) with its updated Binding Cache Entry (u-BCE). Also this mechanism efficiently supports the uplink and downlink packets between mobile nodes, so it avoids superfluous packet forwarding delay and packet loss.
\end{abstract}

Keywords-PMIPv6, transient binding, multi-homing, mLMA, u-BCE, m_MAG, handover

\section{INTRODUCTION}

Proxy mobile IPv6 is a network based mobility management protocol. It is a protocol for building a common and access technology independent of mobile core networks. Proxy Mobile IPv6 is the only network-based mobility management protocol standardized by IETF [1].

In this protocol the host can change its point of attachment without its changing its IP address. Here the mobility need change of COA.(Care of Address)The PMIPv6(Proxy Mobile IPv6) support for this mechanism using m-MAG(modified mobility Access Gateway. There are more than one type of network interfaces are present in the wireless network environment. From this core environment the $\mathrm{MN}$ follows the COA. The transient binding mechanism will support this environment. In the transient binding transient binding cache entry is used to optimize the handover performance for both single and double.[2]
Proxy Mobile IPv6 service and get back the names. Some local mobility anchors may be designated as primary servers and others as backup servers. [3] According to the PMIPv6 base specification, an LMA updates a mobile node's (MN's) Binding Cache Entry (BCE) and switches the forwarding tunnel after receiving a Proxy Binding Update (PBU) message from the mobile node's new MAG (n-MAG). At the same time, the LMA disables the forwarding entry towards the mobile node's previous MAG (p-MAG). In case of an intertechnology handover, the mobile node's handover target interface must be configured according to the Router Advertisement being sent by the n-MAG [2]

According to the PMIPv6 base specification, an LMA updates a mobile node's (MN's) Binding Cache Entry (BCE) and switches the forwarding tunnel after receiving a Proxy Binding Update (PBU) message from the mobile node's new MAG (nMAG). At the same time, the LMA disables the forwarding entry towards the mobile node's previous MAG (pMAG).

One of the primary issues for mobile networking is the multihoming, in which MN has multiple network interfaces, e.g., WLAN and 3G network [4]. However, it is noted that the current PMIPv6 was originally designed without consideration of multi-homing.

For real time transmission, it is essential that packet loss should be reduced or avoided for the user to enjoy high user perceived QoS. Thus, there should be a fast handover binding mechanism to re-route flows to another interface when one interface has lost its connection with the shortest possible delay.

This paper proposes an extensive handover scheme of PMIPv6 with transient binding for multi-homing and mobility support, in which the PMIPv6 Local Mobility Anchor (LMA) will update its binding cache entry (u-BCE) and bind address of the data packets both to the Previous-Mobile Access Gateway (P-MAG) and modified Mobile Access Gateway (m-MAG) toward $\mathrm{MN}$, when $\mathrm{MN}$ is in the handover region. Proxy Mobile IPv6 has One Address (Home Network Prefix). Intelligence in Proxy Mobile IPv6 NW is Local Mobility Anchor (LMA) "Home Agent" and mobile access gateway manages signaling 


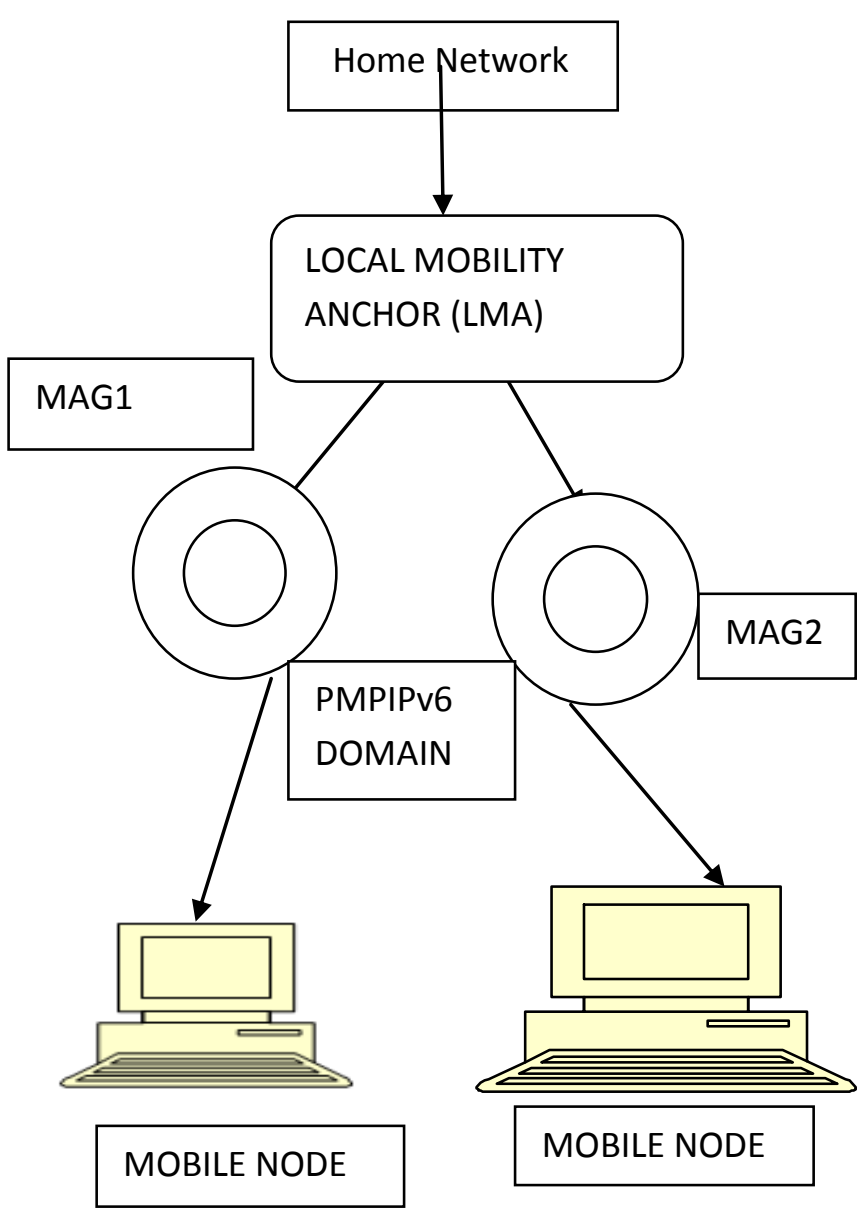

Fig1: Local Mobility Anchor in PMIPv6

According to the PMIPv6 base specification, an LMA updates a mobile node's (MN's) Binding Cache Entry (BCE) and switches the forwarding tunnel after receiving a Proxy Binding Update (PBU) message from the mobile node's new MAG (n-MAG). At the same time, the LMA disables the forwarding entry towards the mobile node's previous MAG (p-MAG). In case of an inter-technology handover, the mobile node's handover target interface must be configured according to the Router Advertisement being sent by the nMAG [2] Figure 1 explains this concept.

\section{MULTIHOMING IN ROXYMOBILE IPv6}

Multi-homing is a situation that describes a single computer host that makes use of several IP addresses associated with various connected networks. Within this scenario, the multihome computer host is physically linked to a variety of data connections or ports. These connections or ports may all be associated with the same network or with a variety of different networks. Depending on the exact configuration, multi-homing may allow a computer host to function as an IP router.

The process of multi-homing makes use of what is known as Stream Control Transmission Protocol, or SCTP. Essentially, the process involves employing multi-homing by making use of a single SCTP endpoint to support the connectivity to more than one IP address. By establishing connection to multiple addresses, multi-homing can help to enhance the overall stability of the connectivity associated with the host.

One of the advantages of multi-homing is that the computer host is somewhat protected from the occurrence of a network failure. With systems that make use of a single IP address and connection, the failure of the connected network means that the connection shuts down, rendering the end system ineffectual as far as connectivity to the Internet is concerned. With multi-homing, the failure of a single network only closes a single open door. All the other doors, or IP addresses associated with the other networks, remain up and functional.

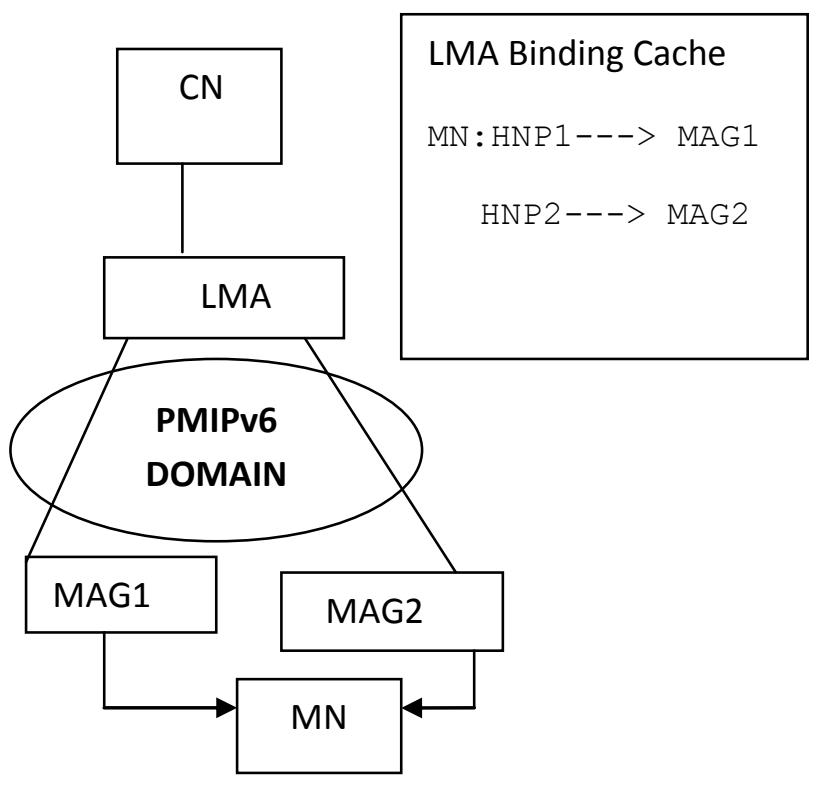

Fig:2. Multihoming Scenario of PMIPv6

\section{TRANSIENT BINDING FOR PROXY MOBILE IPv6}

Transient Binding mechanism solves the problem in hand over by using m-LMA and u-MAG with transient binding cache mechanism.

\subsection{Analysis and Solution of Hand over Problem}

Handovers from a serving network to a target network must be fast so that mobile users continue receiving their services seamlessly. Supporting voice and interactive multimedia with continuous mobility implies that the handover latency should not exceed 50ms to prevent excessive jitter.

Handover in wireless systems is a process to transfer the connection of a Mobile Node (MN) from one point of attachments to another one. During a handover, the MN may experience connectivity interruption and be subject to extra security threats, while mobile users, on the other hand, desire to receive their services seamlessly. In case of real-time interactive services, e.g. VoIP, this requires containing the overall IP-level handover latency - the time interval in which the MN does not send or receive IP packets - within 50ms to prevent excessive jitter [1][5]. Unfortunately, the vast majority of the handovers do not currently meet this goal due to the latencies associated with packet loss and signaling and reconfiguration overhead [6] 
When MN changes its point of attachment, the P-MAG will detect the MN's detachment and will perform the Proxy Biding Update (PBU) operations with the LMA to remove the binding and routing states associated with MN. Upon receiving this PBU (DeReg PBU) request, the LMA will identify the corresponding $\mathrm{BCE}$ entry and accept the request. After that, it expects to receive the PBU message of the NMAG for some time. In this period, a certain amount of handover latency occurs. During this handover period, some packet losses may occur. Accordingly, it is not easy to achieve seamless handover.[2]

This document specifies transient BCEs [2] as an extension to the PMIPv6 protocol. Set up and configuration of a transient BCE can be performed by means of extended PMIPv6 signaling messages between the MAG and the A component using a new Transient Binding mobility option.

The transient BCE mechanism supports three clearly distinguished sequences of transient states to suit various handover scenarios and to improve handover performance for both inter- and intra-technology handover. As a result of using transient BCEs, excessive packet buffering at the m-MAG during the MN's handover process is not necessary and packet losses and major jitter can be avoided. The Transient Binding option has no alignment requirement. Its format is given in figure3.
This section describes the format of the Transient Binding option, which can be included in a Proxy Binding Update message and a Proxy Binding Acknowledge message. The use of this Mobility Header option is optional. The Transient Binding option can be included in a PBU message, which is sent by an MN's m-MAG as a result of a handover. In such a case, the m-MAG controls the transient BCE on the LMA. Alternatively, the LMA may attach the Transient Binding option in a PBA for two reasons. Either it replies to a received PBU with an attached Transient Binding option to approve or correct the transient BCE lifetime, or it notifies the m-MAG about its decision to enter a transient BCE without having received a Transient Binding option from the m-MAG in the associated PBU beforehand.

Type: Identifies the Transient Binding option.

L-Flag: Indicates that the LMA applies late path switch according to the transient BCE state. If the L-flag is set to 1, the LMA continues to forward downlink packets towards the p-MAG. Different setting of the L-Flag may be for future use. Length: 8-bit unsigned integer indicating the length of the option in octets, excluding the Type and the Length fields. This field MUST be set to 2. Lifetime: Maximum lifetime of a Transient-L state in multiple of $100 \mathrm{~ms}$

012345678901234567890123456789012345678901234567

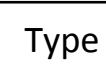

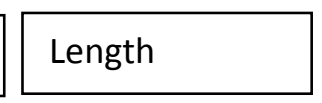

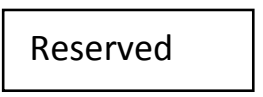

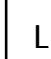

Life Time

Figuer3: Format of Transient binding for proxy Binding Update

\section{Support of Transient Binding for Reducing Hand over Latency and Packet Loss}

Multi-homing technology handle the concept handover with multiple interfaces. In that the PMIPv6 have the problem of message format which is solved by the transient binding.[2] Because of this transient binding multiple interface problem solved using automatic address configuration which is available in Proxy mobile IPv6[7].But without the transient binding the automatic address configuration provide only one PBU for that particular CoA of that MN, so the MN not able to identify the U-PBU so there is lag in the hand over, because this lag there is also pocket loss problem is arise. These draw back of the PMIPv6 is solved by the extension of multi-homing with transient binding.
Figure 4 explains the concept of possible transient forwarding states during a handover. In this with time the current state of LMA i.e. m-LMA and u-BCE is also identified.

When $\mathrm{MN}$ connects to the new link, it establishes a physical link connection with N-MAG (for example, radio channel assignment), which in turn triggers the establishment of a link-layer connection with the N-MAG. An IP layer connection setup may be performed at this time. This step can be a substitute for Unsolicited Neighbor Advertisement (UNA) in [11]. 
BEFORE HAND

OVER

\section{P-MAG, (with uplink and downlink) PBU ( $m-M A G$ and PBA (LMA)}

\section{Transient Hand over Procedure}

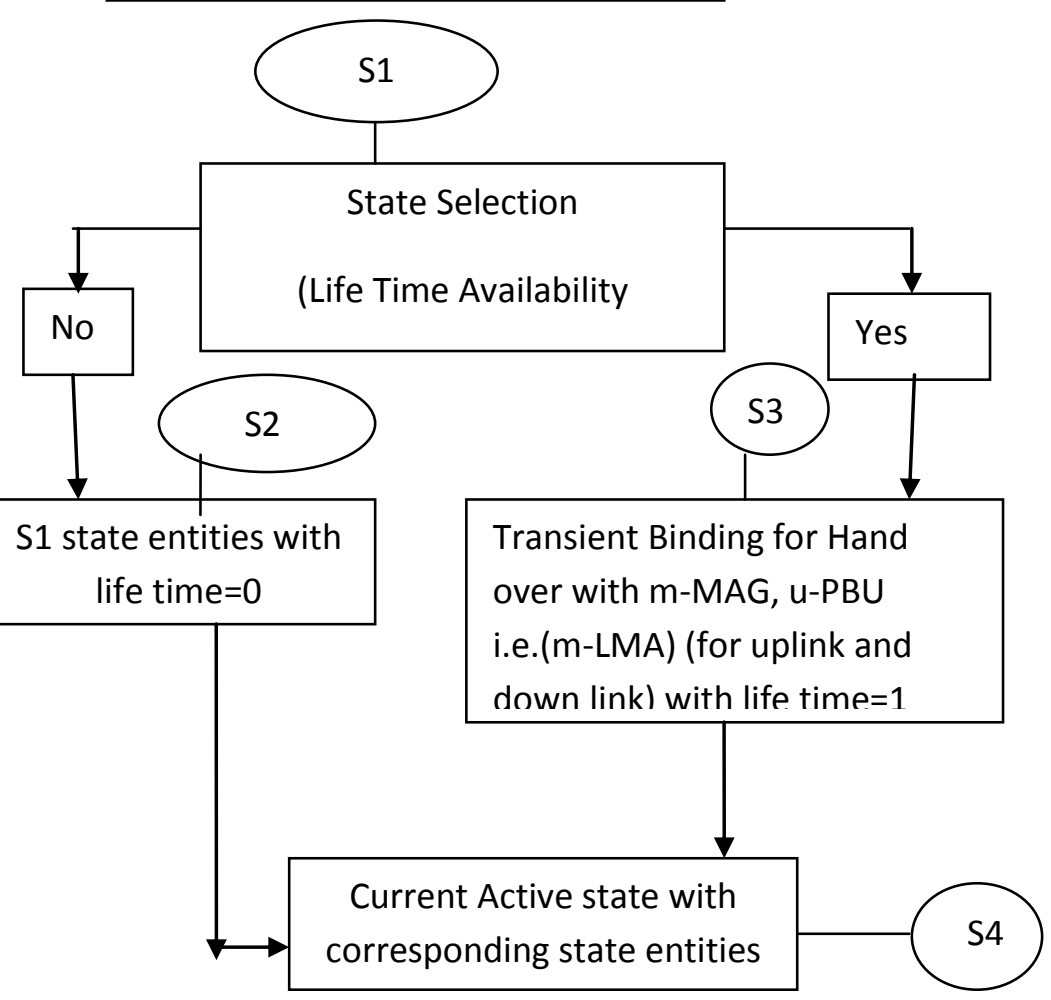

Figure 4: Possible transient forwarding states during a handover

In the given figure4: $\mathrm{S} 2, \mathrm{~s} 3, \mathrm{~s} 4$ specifies the mechanism of transient binding with its entities Mn,LMA,MAG.s4 specifies the total transient binding of PMIPv6 with the extension multi-homing mechanism

First, when MN moves to the transient binding region, it detects that a handover is imminent, and thus it performs the link layer signaling. The P-MAG sends Handover Init (HI) message to N-MAG, where HI messages includes the MN's IP addresses that are Proxy-CoA (P-CoA), Home address (MN-HoA), LMA address (LMAA), MN's Identifier, and HNP. When the m-MAG receives HI message, it should examine whether a tunnel to LMA exists or not. If the tunnel has not been established, it should establish the tunnel with LMA. To establish the tunnel, the N-MAG sends a PBU message to LMA, which includes MN Identifier, MN-HoA, and HNP.

When LMA receives the PBU message, it creates a new binding cache entry or modifies the existing binding cache entry [5][2]. If the LMA successfully processes the PBU it sets the tunnel with m-MAG to send and receive the data packets. After successful establishment of the tunnel, the
LMA sends a PBA message to indicate whether or not the PBU message was processed successfully. If there is a failure, the PBA message indicates the failure. Otherwise, m-MAG creates a tunnel to LMA and ensures that the data packets with the destination address as Proxy CoA are copied and forwarded to LMA over the tunnel. It also creates a host route for forwarding packets to the MN. When MN connects to the new link, it establishes a physical link connection with mMAG (for example, radio channel assignment), which in turn triggers the establishment of a link-layer connection with the m-MAG. An IP layer connection setup may be performed at this time. This step can be a substitute for Unsolicited Neighbor Advertisement (UNA) in [11]. Then, the m-MAG sends a Handover ACK message back to the P-MAG to indicate whether the handover procedure was successfully done or not. The m-MAG sends a PBU (De-registration) message to LMA. This message includes MN-identifier and P-CoA of m-MAG. On reception of this PBU message, the LMA deletes the HNP1 of IF1 in the binding cache entry and release the tunnel between LMA and P-MAG). In response to PBU (Deregistration) message, the LMA sends PBA message to $\mathrm{m}-\mathrm{MAG}$

For real time transmission, it is essential that packet loss should be reduced or avoided for the user to enjoy high user perceived QoS. Thus, there should be a fast handover binding mechanism to re-route flows to another interface when one interface has lost its connection with the shortest possible delay.[4]

A possible approach to solve this issue will be such that a transient binding to flows tied to an interface via which disconnection will happen to a stable interface needs to be present and stored in the system whereby, when disconnection happens packet loss can be prevented. Such mechanism is highlighted in this paper

\subsection{Simulation Results and Comparison}

In order to further analyze (Transient binding PMIPv6 is represented as T-PMIPv6) T-PMIPv6 and compare its performance to PMIPv6 and F-PMIPv6, it is decided to simulate the three protocols and acquire the results in a similar fashion as the mathematical model. The protocols have been simulated used Network Simulator (NS2). NS2 is an event simulator targeting network research and has a support for many protocols over the different network layers.

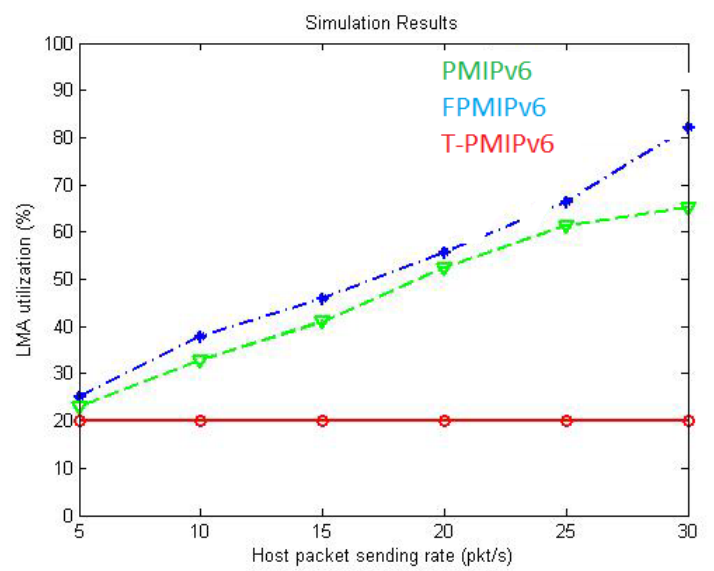




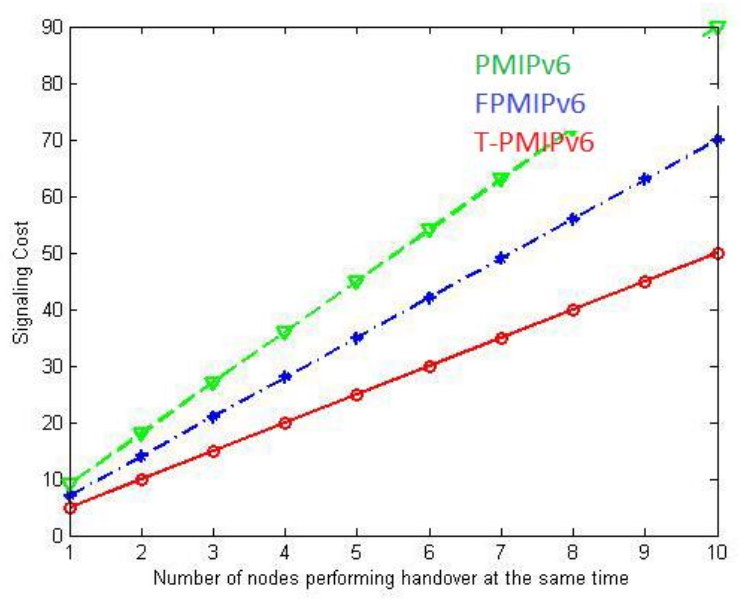

\section{CONCLUSION}

This paper proposed an extension handover scheme of the PMIPv6 with transient binding for multi-homing and mobility, in which the PMIPv6 binding update is performed in advance and then LMA performs the binding and the data packets to the m-MAG as well as P-MAG. The LMA is extended to support the multiple binding cache entry (BCE).

This mechanism offers binded data to the MN's new access router that shortens the handover latency period and decreases packet loss. It showed that buffering could eliminate the packet loss. This gives sufficient time for security subprocesses like mutual MN-network authentication, authorization and key distribution. We showed that when the handover prediction time is not sufficient, the protocol still functions properly and its performance converges towards that of PMIPv6

The proposed work highlighted additional work that has to be done with respect to multi-homing for the PMIPv6 protocol. The main categories of additional are, Achieving flow mobility when a subset of prefixes needs to be transferred to the newly powered on interface or connected interface. Extending PMIPv6 operation in scenarios where multiple interfaces are attached to the same MAG. Achieving flow mobility when a subset of prefixes needs to be transferred to the newly powered on interface or connected interface.

\section{REFERENCES}

[1] D. Johnson, C. Perkins, J. Arkko, "Mobility Support in IPv6”, IETF RFC 3775, June 2004.

[2] M. Liebsch, A. Muhanna, O. Blume, "Transient Binding for Proxy Mobile IPv6", IETF Internet Draft draft-ietf-mipshop-transient-bcepmipv6-06, July 2010.

[3] B. Sarikaya, F. Xia, "Local Mobile Anchor Discovery Using DNS by Service Name" IETF Internet Draft draftsarikaya-netlmm-lma-dnsdiscovery-01,July 13

[4] M. Jeyatharan, C. Ng, "Multihoming Problem Statement in NetLMM", IETF Internet Draft draft-jeyatharannetext-multihomingps- 02, March 2010.

[5] RFC4140, "Hierarchical Mobile IPv6 Mobility Management (HMIPv6)", IETF, August 2005.

[6] A. Mishra, M. Shin and W. A. Arbaugh, "Pro-active Key Distribution Using Neighbor Graphs", IEEE Wireless Comm. Magazine, vol.11 no.1, February 2004, pp.26-36.

[7] Gundavelli, S., Leung, K., Devarapalli, V., Chowdhury, K., and B. Patil, "Proxy Mobile IPv6", RFC 5213, August 2008

[8] Jeyatharan, M., Ng, C., Gundavelli, S., Leung, K., and V.Devarapalli, "Partial Handoff Support in PMIPv6", draft-jeyatharan-netext-pmip-partial-handoff-02), February 2010

[9] Koodli, R. and K. Chowdhury, "Flow Handover for Proxy Mobile IPv6", draft-koodli-netext-flow- andover01 (work in progress), October 2009

[10] Hui, M. and H. Deng, "PMIPv6 Multihoming Extension and Synchronization in LMA and MAG", draft-huinetext-multihoming-00 October 2009.

[11] R. Koodli, Ed., "Mobile IPv6 Fast Handovers", IETF RFC 5568, July 2009 